\title{
Postoperative delirium in the elderly: diagnosis and management
}

\section{Thomas N Robinson Ben Eiseman}

Department of Surgery, University of Colorado at Denver Health Sciences Center, Aurora, CO, USA

Correspondence:Thomas Robinson 12631 East 17th Ave., Mail Stop C313, PO Box 65II, Aurora, CO 80045, USA $\mathrm{Tel}+\mathrm{I} 3037242728$

Fax +l 3037242733

Email thomas.robinson@uchsc.edu

\begin{abstract}
Delirium is a common but often undiagnosed complication in the elderly following a major operation. Recognizing the presentation of delirium and the criteria to establish the diagnosis of delirium will improve a clinician's ability to detect this complication. Treating delirium with environmental, supportive, and pharmacologic interventions reduces the incidence and side effects of postoperative delirium. The purpose of this review is to describe the diagnosis and treatment of postoperative delirium.
\end{abstract}

Keywords: postoperative, delirium, geriatric, elderly, review

\section{Introduction}

Perioperative complications specific to elderly patients are becoming increasingly relevant with an aging population (Etzioni et al 2003). Postoperative delirium is a quintessential geriatric complication (Inouye et al 2007). The incidence ranges from $9 \%$ to $87 \%$ depending on both the patient population and the degree of operative stress (Demeure and Fain 2006). The recognition and treatment of delirium is critically important because postoperative delirium is associated with poor outcomes including functional decline, longer hospitalization, institutionalization, greater costs, and higher mortality (Dasgupta and Dumbrell 2006). The purpose of this review is to describe the diagnosis and treatment of postoperative delirium.

\section{Risk factors}

There are multiple risk factors for developing postoperative delirium. Risk factors can be separated into patient-specific and operation-specific risk factors.

Established patient-specific risk factors for the development of delirium following noncardiac operations include pre-existing dementia, older age, functional impairment, greater co-morbidities, and psycopathological symptoms (Dasgupta and Dumbrell 2006) (see Figure 1A). Assessing all preoperative risk factors, pre-existing dementia appears to be the strongest predictor for the occurrence of postoperative delirium (Dasgupta and Dumbrell 2006).

Operation-specific risk factors for the development of postoperative delirium are based on the degree of operative stress. For example, low operative stress procedures such as cataract surgery result in delirium in $4 \%$ of cases (Milstein et al 2002) in comparison to high surgical risk procedures such as vascular operations which result in delirium in $36 \%$ of cases (Marcantonio et al 2002).

The risk factors for developing delirium are additive (Marcantonio et al 1994); therefore, recognizing a patient with multiple risk factors for delirium should trigger the surgeon to implement environmental and supportive measures that have been proven to prevent the onset and shorten the duration of delirium (Inouye 2006) (see Figure 1B). 


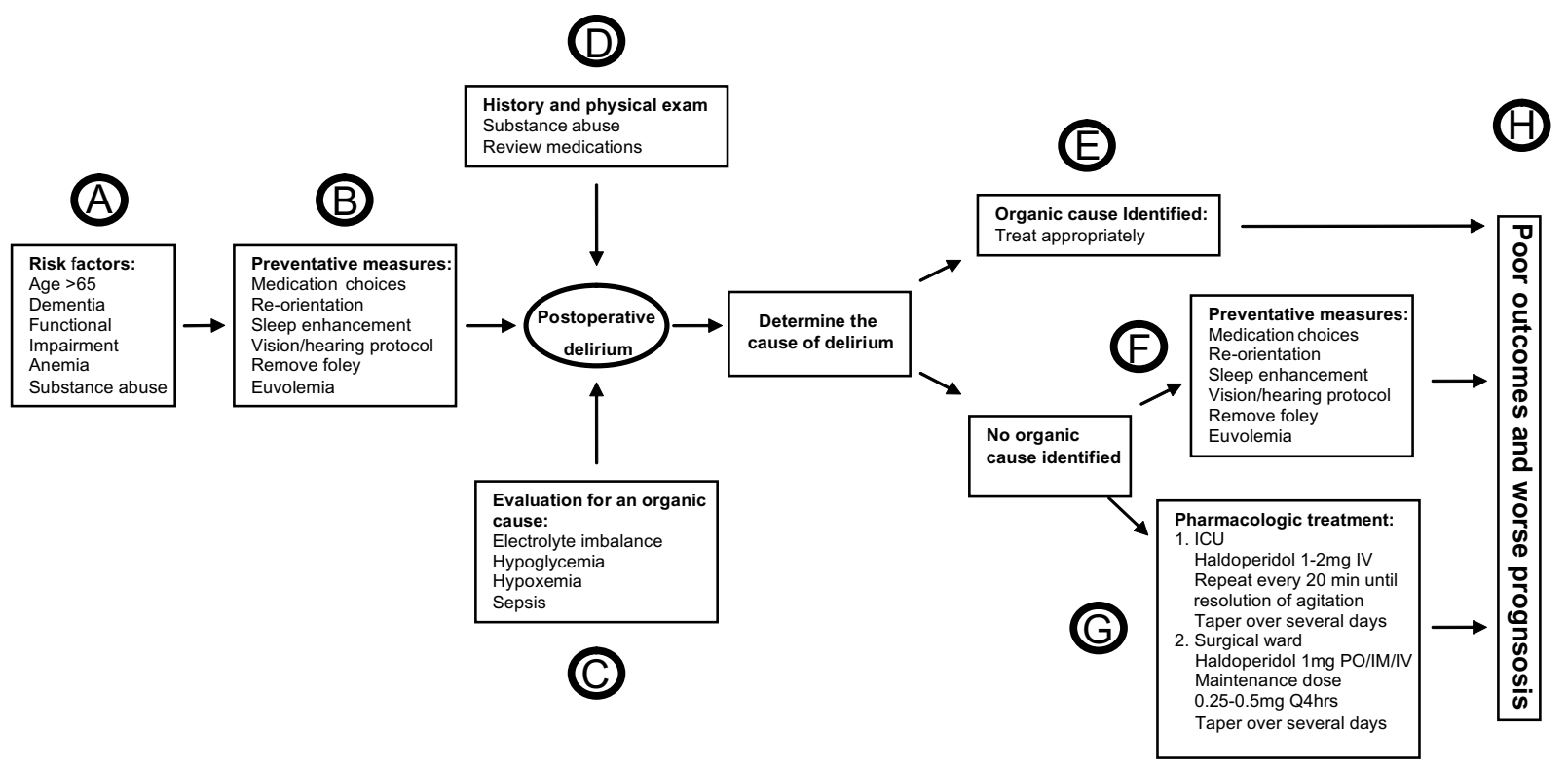

Figure I Postoperative delirium in the elderly - A diagnostic and treatment algorithm.

\section{Diagnosis}

Delirium is defined as an acutely altered and fluctuating mental status with features of inattention and an altered level of consciousness. Post-operative cognitive dysfunction should be distinguished from delirium and is defined as a disorder in thought processes which effect cognition in terms of memory, comprehension, and attention (Bryson and Wyand 2006). Over $50 \%$ of delirium on inpatient services is unrecognized by the clinical team (Milisen et al 2002). Understanding the three motor types of delirium helps the clinician to recognize the presence of delirium (Meagher and Trzepacz 2000). First, hyperactive delirium occurs when a patient is restless, irritable, combative, or agitated. Second, hypoactive delirium presents with lethargy, decreased alertness/motor activity, and unawareness. And third, the mixed subtype of postoperative delirium has characteristics of both hypoactive and hyperactive delirium. The incidences of the motor subtypes of postoperative delirium are: hypoactive $71 \%$, mixed $29 \%$, and hyperactive is rare (Marcantonio et al 2002).

The diagnosis of delirium is established through using existing delirium assessment methods. The Confusion Assessment Method-Intensive Care Unit (CAM-ICU) is a straight-forward tool with established reliability and validity for assessing delirium (Ely et al 2001). The CAM-ICU combines an assessment of the patient's sedation or level of consciousness with an evaluation of mental status, inattention, disorganized thinking, and an altered level of consciousness (Ely et al 2001). To assess the level of sedation, the CAM-ICU utilizes the Richmond Agitation and Sedation Score which is a 10 point scale that provides discrete criteria for levels of sedation and agitation (Ely et al 2003). The benefit of the CAM-ICU is that it provides a brief and easy delirium assessment tool that can be administered by both physicians and nurses (McNicoll et al 2005). The CAMICU should be performed daily on all patients who have multiple risk factors (older than 65 years, cognitive dysfunction, multiple co-morbidities) for developing postoperative delirium.

The Mini-Mental State Examination (MMSE) is another tool for assessing cognitive status (Folstein et al 1975). The MMSE measures orientation, attention, calculation, recall, and language, which allows both for screening cognitive dysfunction and following fluctuations over time. Other bedside tests to determine the presence of delirium include the executive clock drawing task, the Informant Questionnaire on Cognitive Decline in the Elderly and the Memorial Delirium Assessemnt Scale (Moraga and Rodriguez-Pascual 2007).

\section{Evaluation}

After the diagnosis of delirium has been established, a standardized work-up to exclude organic or identifiable causes of delirium is necessary. The initial step of the evaluation is a complete history and physical exam with cognitive assessment specific for delirium. Further work-up requires the surgeon to tailor their evaluation to an individual patient but can be broken into four main areas:

\section{Laboratory evaluation}

Several reversible metabolic causes of delirium are readily diagnosed with standard laboratory tests. Routine blood 
work includes: electrolytes, glucose, arterial blood gas, calcium, phosphate and magnesium. Correction of the underlying abnormality is completed to reverse the delirium. (see Figure 1C).

\section{Infection work-up}

Evaluation for infection is essential in the postoperative patient. Urosepsis (urine analysis), pneumonia (chest X-ray), line sepsis/bacteremia (blood cultures), and procedurespecific infection (imaging surgical site) are the most common types of infections. In the older patient, occult infections present without the usual physiologic response of a younger adult such as fever and leukocytosis; requiring vigilance by the surgeon to pursue an occult infectious source. Expeditious treatment of the underlying infection is performed medically with antibiotics and when appropriate surgically with drains or re-exploration (see Figure 1C).

\section{Review medication list}

Drug induced post-operative delirium is common. Drugs that promote central anticholinergic activity are the most common culprits. Commonly used medications in the postoperative period which cause delirium include: cimetidine, corticosteroids, diphenhydramine, belladonna, promethazine, warfarin, narcotics, benzodiazepines, and antiparkinsonian drugs (Demeure and Fain 2006). The older patient's postoperative medication orders should be tailored to avoid these medications, or when necessary, utilize lower dosages. The aging brain is more susceptible to developing delirium when exposed to anesthetic agents; the most common three medication which result in delirium are benzodiazepines, morphine, and anticholinergics (Young and Inouye 2007) (see Figure 1D).

\section{Substance abuse}

A careful review of the patient's history of substance abuse is required. Withdrawal from alcohol or other illicit substances requires specific treatment and must not be confused with other types of delirium in the elderly postoperative patient. Former alcoholics should receive thiamine, which is the key drug for the management of Korsadoff's psychosis (see Figure 1D).

\section{Imaging}

Routine imaging is not a mandatory component of the evaluation of delirium. Computed tomography of the head is indicated when neurological deficits are found on physical exam suggesting stroke or when an extensive search for other etiologies of delirium has failed to define a cause (Amador and Goodwin 2005).

\section{Treatment}

The treatment of delirium requires a multicomponent strategy aimed at both optimizing preventative measures in addition to pharmacologic management (Trzepacz et al 1999). Delirium prevention should start in the operating room by maintaining hemodynamic stability, providing adequate oxygenation, optimizing the acid base status, minimizing electrolyte abnormalities and administering appropriate drug dosages during the operation (Jin and Chung 2001). Prior to implementing any treatment strategy, assessment for an underlying organic cause must be performed because specific treatment for the underlying diagnoses would differ from the management of nonspecific postoperative delirium in the geriatric patient (see Figure 1E).

Implementing preventive measures, including both environmental and supportive interventions, to avoid delirium has been shown to reduce both the number and duration of delirium episodes in elderly hospitalized patients (see Figure 1B). Specific interventions which have been shown to reduce delirium include: (1) an orientation protocol to provide the patient with repeated orientation to their surroundings and care team members, (2) a sleep protocol to provide uninterrupted night time sleep, (3) an early-mobilization protocol to allow for daily ambulation and range of motion, (4) a vision protocol to allow easy access to glasses and other visual aids, and (5) a hearing protocol to provide amplifying devices and other hearing aids (Inouye et al 1999). These environmental and supportive protocols reduced the incidence of delirium from $15 \%$ to $10 \%$ with a reduction in both duration and total episodes of delirium but did not decrease hospital length of stay (Inouye et al 1999). Other environmental and supportive strategy studies which reduce the incidence of delirium have also been shown to reduce complication related to delirium including decubitus ulcers, urinary tract infections, nutritional complications, sleeping problems, and falls (Lundstrom et al 2007).

Specific care should be taken to avoid dehydration or hypovolemia which exacerbates delirium. Nonessential catheters such as foleys, nasogastric tubes, or multiple intravenous access lines should be avoided because their presence is linked to an increased risk of delirium.

Safety issues must be addressed for patients with risk of self-harm. In patients with mixed motor or hyperactive delirium, agitation may lead to inadvertently pulled tubes, drains or lines. Hypoactive delirium can result in inadvertent 
wandering. All delirium motor types are a risk for falls. Judicious clinical judgment and communication with the nursing team are essential for determining whether a sitter or increased acuity to the step down unit or ICU may be warranted. Restraints should be used when other means of treating delirium have failed as restraints can exacerbate delirium and lead to injuries (Trzepacz et al 1999). Family members should be encouraged to interact with the delirious patient as their input helps maintain adaptive cognitive functioning (Trzepacz et al 1999).

Pharmacologic treatment of delirium is required in many elderly patients following major operations. Neuroleptic agents, particularly haloperidol, are the medication of choice to treat delirium (Kalisvaart et al 2005; Inouye 2006) (see Figure 1G). Prospective placebo controlled data has established the superiority of haloperidol over benzodiazepines (Breitbart et al 1996). Atypical antipsychotics, most notably respiridone, have been compared with haloperidol for the treatment of delirium, but have not shown superiority (Han and Kim 2004). Dosing recommendations vary depending on the severity of the delirium as well as clinical setting of the ICU or surgical ward.

\section{Pharmacologic treatment for postoperative delirium in the ICU}

For delirium in the ICU, a loading dose of $2 \mathrm{mg}$ of intravenous haloperidol is administered with repeated doses every 15 to 20 minutes while the agitation persists (Jacobi et al 2002). If the agitation is severe, doubling the dose of haloperidol every 15 to 20 minutes is acceptable. After the delirium is controlled, scheduled haloperidol is prescribed over the next few days to avoid relapses of severe agitation (Jacobi et al 2002).

\section{Pharmacologic treatment for postoperative delirium in the surgical ward}

Haloperidol can be administered orally, intramuscularly, and intravenously. An initial dose of 1 to $2 \mathrm{mg}$ of haloperidol is recommended with doses of 0.25 to $0.5 \mathrm{mg}$ every 4 hours for maintenance dosing in elderly patients (Trzepacz et al 1999). For patients with persistent agitation, titration to higher dosages of haldoperidol is acceptable (Trzepacz et al 1999).

Potential side effects due to neuroleptic treatment require monitoring. The two most common side effects of haloperidol include a prolonged QT-interval on the electrocardiogram and extrapyramidal side effects (Jacobi et al 2002). Patients receiving haloperidol should have their QT-interval monitored by electrocardiogram (Trzepacz et al 1999; Jacobi et al 2002). Adverse side effects may be managed by lowering the dosage of haldoperidol or discontinuing the medication (Jacobi et al 2002).

The surgeon's awareness of the clinical risk factors for the development of delirium should influence the decision to admit a patient after what is typically considered an outpatient procedure. Recognizing the risk factors which make a patient more vulnerable to developing postoperative delirium should result in an inpatient observation rather then same day discharge for frail subjects undergoing operations such as laparoscopic cholecystectomy or orthopedic surgery after which a younger healthy individuals are discharged home.

Other less common etiologies of delirium exist which require specific treatment plans. First, sedative or analgesic withdrawal results in delirium and occurs in patients who are exposed to as little as one week of high dose sedative or analgesic therapy. The recommended treatment of delirium resulting from cessation of sedatives or analgesics is performing a systematic taper and starting an alpha-2 agonist which reduces concurrent sedative and analgesic requirements (Jacobi 2002). Second, profound delirium can occur postoperatively due to the central anticholinergic syndrome (Katsanoulas et al 1999). The central anticholinergic syndrome is caused by anticholinergic medications that cross the blood-brain barrier and block muscarinic cholinergic receptors resulting in dramatic delirium which can be hyperactive or hypoactive. The recommended treatment of suspected central anticholinergic syndrome is the cholinesterase inhibitor physostigmine dosed at 10-30 micrograms/kilogram intravenously (Moos 2007).

\section{Outcomes}

Delirium is a critically important complication in the elderly because it results in functional decline, longer hospitalization, and institutionalization (Dasgupta and Dumbrell 2006) (see Figure $1 \mathrm{H}$ ). Complications specific to delirium include falls, pulled lines/tubes, aspiration pneumonia, and increased use of bladder catheters (Demeure and Fain 2006). Recent studies have suggested a link between delirium in the critically ill and long term cognitive dysfunction through a mechanism hypothesized as delirium acting as a subclinical dementing illness (Hopkins and Jackson 2006). Patients who develop delirium during their hospitalization have a higher six month mortality in comparison with patients who do not develop delirium (Ely et al 2004). 


\section{Summary}

Delirium is a common but often undiagnosed complication in the elderly following a major operation. Recognizing the presentation of delirium and the criteria to establish the diagnosis of delirium will improve a clinician's ability to detect this complication. Delirium can be diagnosed with ease and brevity with delirium assessment tools such as the CAM-ICU. Initial treatment of delirium should be with environmental and supportive measures which can be instituted as preventative in patients with multiple risk factors. Subsequently, pharmacologic treatment is implemented. Optimal treatment of postoperative delirium reduces the incidence, duration, and side effects of this common complication in geriatric postoperative patients.

\section{Disclosure}

This work was supported by a Geriatrics Education for Specialty Residents (GSR) grant from the American Geriatrics Society and the John A Hartford Foundation.

\section{References}

Amador LF, Goodwin JS. 2005. Postoperative delirium in the older patient. J Am Coll Surg, 200:767-73.

Breitbart W, Marotta R, Platt MM, et al. 1996. A double-blind trial of haloperidol, chlorpromazine, and lorazepam in the treatment of delirium in hospitalized AIDS patients. Am J Psychiatry, 153:231-7.

Bryson GL, Wyand A. 2006. Evidence-based clinical update: general anesthesia and the risk of delirium and postoperative cognitive dysfunction. Can J Anaesth, 53:669-77.

Dasgupta M, Dumbrell AC. 2006. Preoperative risk assessment for delirium after noncardiac surgery: a systematic review. J Am Geriatr Soc, 54:1578-89.

Demeure MJ, Fain MJ. 2006. The elderly surgical patient and postoperative delirium. J Am Coll Surg, 203:752-7.

Ely EW, Inouye SK, Bernard GR, et al. 2001. Delirium in mechanically ventilated patients: validity and reliability of the confusion assessment method for the intensive care unit (CAM-ICU). JAMA, 286:2703-10.

Ely EW, Shintani A, Truman B, et al. 2004. Delirium as a predictor of mortality in mechanically ventilated patients in the intensive care unit. JAMA, 291:1753-62.

Ely EW, Truman B, Shintani A, et al. 2003. Monitoring sedation status over time in ICU patients: reliability and validity of the Richmond Agitation-Sedation Scale (RASS). JAMA, 289:2983-91.

Etzioni DA, Liu JH, Maggard MA, et al. 2003. The aging population and its impact on the surgery workforce. Ann Surg, 238:170-7.

Folstein MF, Folstein SE, McHugh PR. 1975. "Mini-mental state". A practical method for grading the cognitive state of patients for the clinician. $J$ Psychiatr Res, 12:189-98.
Han CS, Kim YK. 2004. A double-blind trial of risperidone and haloperidol for the treatment of delirium. Psychosomatics, 45:297-301.

Hopkins RO, Jackson JC. 2006. Assessing neurocognitive outcomes after critical illness: are delirium and long-term cognitive impairments related? Curr Opin Crit Care, 12:388-94.

Inouye SK. 2006. Delirium in older persons. N Engl J Med, 354:1157-65.

Inouye SK, Bogardus ST Jr., Charpentier PA, et al. 1999. A multicomponent intervention to prevent delirium in hospitalized older patients. $N$ Engl $J$ Med, 340:669-76.

Inouye SK, Studenski S, Tinetti ME, et al. 2007. Geriatric syndromes: clinical, research, and policy implications of a core geriatric concept. $J$ Am Geriatr Soc, 55:780-91.

Jacobi J. 2002. Clinical practice guidelines for the sustained use of sedatives and analgesics in the critically ill adult. Am J Health Syst Pharm, 59:150-78.

Jacobi J, Fraser GL, Coursin DB, et al. 2002. Clinical practice guidelines for the sustained use of sedatives and analgesics in the critically ill adult. Crit Care Med, 30:119-41.

Jin F, Chung F. 2001. Minimizing perioperative adverse events in the elderly. Br J Anaesth, 87:608-24.

Kalisvaart KJ, de Jonghe JF, Bogaards MJ, et al. 2005. Haloperidol prophylaxis for elderly hip-surgery patients at risk for delirium: a randomized placebo-controlled study. J Am Geriatr Soc, 53:1658-66.

Katsanoulas K, Papaioannou A, Fraidakis O, et al. 1999. Undiagnosed central anticholinergic syndrome may lead to dangerous complications. Eur J Anaesthesiol, 16:803-9.

Lundstrom M, Olofsson B, Stenvall M, et al. 2007. Postoperative delirium in old patients with femoral neck fracture: a randomized intervention study. Aging Clin Exp Res, 19:178-86.

Marcantonio E, Ta T, Duthie E, et al. 2002. Delirium severity and psychomotor types: their relationship with outcomes after hip fracture repair $J$ Am Geriatr Soc, 50:850-7.

Marcantonio ER, Goldman L, Mangione CM, et al. 1994. A clinical prediction rule for delirium after elective noncardiac surgery. JAMA, 271:134-9.

McNicoll L, Pisani MA, Ely EW, et al. 2005. Detection of delirium in the intensive care unit: comparison of confusion assessment method for the intensive care unit with confusion assessment method ratings. $J \mathrm{Am}$ Geriatr Soc, 53:495-500.

Meagher DJ, Trzepacz PT. 2000. Motoric subtypes of delirium. Semin Clin Neuropsychiatry, 5:75-85.

Milisen K, Foreman MD, Wouters B, et al. 2002. Documentation of delirium in elderly patients with hip fracture. J Gerontol Nurs, 28:23-9.

Milstein A, Pollack A, Kleinman G, et al. 2002. Confusion/delirium following cataract surgery: an incidence study of 1-year duration. Int Psychogeriatr, 14:301-6.

Moos DD. 2007. Central anticholinergic syndrome: a case report. J Perianesth Nurs, 22:309-21.

Moraga AV, Rodriguez-Pascual C. 2007. Acurate diagnosis of delirium in elderly patients. Curr Opin Psychiatry, 20:262-7.

Trzepacz P, Breitbart W, Franklin J, et al. 1999. Practice guideline for the treatment of patients with delirium. American Psychiatric Association. Am J Psychiatry, 156:1-20.

Young J, Inouye SK. 2007. Delirium in older people. BMJ, 334:842-6. 
